\title{
TI.25.1
}

(1) Document Has Been Moved

The Grouper Deployment Guide is now available in the wiki at Grouper Deployment Guide. The previous version is archived here in the document repository below.

\section{TIER Grouper Deployment Guide}

- PDF: TI.25.1-TIERGrouperDeploymentGuide.pdf

- Text: TI.25.1-TIERGrouperDeploymentGuide-TEXT.txt

\section{More Information}

\begin{tabular}{|c|c|}
\hline Repository ID & TI.25.1 \\
\hline Persistent URL & http://doi.org/10.26869/TI.25.1 \\
\hline Title & TIER Grouper Deployment Guide \\
\hline Authors & James Babb, Tom Dopirak, Bill Thompson, TIER API and Entity Registry WG, Grouper Development Team \\
\hline Sponsor & Internet2 \\
\hline \multicolumn{2}{|l|}{ Review } \\
\hline Status & Deprecated \\
\hline Publish Date & $4 / 21 / 2017$ \\
\hline DOI & 10.26869/TI.25.1 \\
\hline \multicolumn{2}{|l|}{ Signature } \\
\hline Deprecated & No \\
\hline Future Review & April 2018 \\
\hline \multicolumn{2}{|l|}{ Supersedes } \\
\hline Format & PDF \\
\hline \multicolumn{2}{|l|}{ Related Docs } \\
\hline \multicolumn{2}{|c|}{ Development Location } \\
\hline IP Framework & CC BY 4.0 \\
\hline Subject Tags & $\begin{array}{l}\text { Grouper, access management, authorization, access control, access control } \\
\text { model, access control policy }\end{array}$ \\
\hline Notes & see Grouper Deployment Guide for current version \\
\hline
\end{tabular}

